RAL-TR/95-048

\title{
Unitarity and the Time Evolution of Quantum Mechanical States
}

\author{
P.K. Kabirf and A. Pilaftsisin \\ Rutherford Appleton Laboratory, Chilton, Didcot, Oxon, OX11 OQX, UK \\ and \\ Institute of Nuclear and Particle Physics, J.W. Beams Laboratory of Physics, \\ University of Virginia, Charlotessville, VA 22901, USA
}

\begin{abstract}
The basic requirement that, in quantum theory, the time-evolution of any state is determined by the action of a unitary operator, is shown to be the underlying cause for certain "exact" results which have recently been reported about the time-dependence of transition rates in quantum theory. Departures from exponential decay, including the "Quantum Zeno Effect", as well as a theorem by Khalfin about the ratio of reciprocal transition-rates, are shown to follow directly from such considerations. At sufficiently short times, unitarity requires that reciprocity must hold, independent of whether $T$-invariance is valid. If $T$-invariance does not hold, unitarity restricts the form of possible time-dependence of reciprocity ratios.
\end{abstract}

\footnotetext{
*E-mail address: pkk@physics.virginia.edu

†E-mail address: pilaftsis@v2.rl.ac.uk
} 


\section{Introduction}

The Weisskopf-Wigner theory [1] of decaying states has been used with great success in a wide variety of applications. Nevertheless, since it is an approximate theory, it is not surprising that there should be circumstances in which one expects [2, 3, 4] departures from the predictions of the theory. Some of these issues have acquired renewed interest because of advances in experimental methods [5]; others arise from the expected [6, 7] deviation from time-reversal symmetry in weak interactions. The question of the correct treatment of unstable particles also arises in the application of current gauge theories of weak interactions, where the instability of intermediate bosons and fermions cannot always be neglected [8]. In this note, we show that many of these corrections can be directly traced back to the fundamental requirement of unitarity, which is satisfied only approximately in the Weisskopf-Wigner method.

In the Weisskopf-Wigner approximation — which can be generalized [9] to the case of decays arising from two or more states - certain initial states are singled out for special attention. Transitions from these distinguished states, to other states, deplete the population of these initial states. The Weisskopf-Wigner approximation allows for this by replacing the matrix-elements of the exact (full) Hamiltonian, in the subspace spanned by those states, by a non-Hermitian submatrix. For a single unstable state, the (negative) imaginary part of the complex "energy" assures that the probability decreases exponentially with time. While this prescription accounts for the "leakage" of probability in terms of the rate of transitions out of the initial states, detailed analysis outlined below, shows that the Weisskopf-Wigner procedure cannot satisfy unitarity exactly. The circumstance [2] that the "law" [10] of exponential decay cannot be exactly right in quantum theory can be directly related to the fact that the exponential Ansatz is incompatible with the unitarity requirement which is essential for the basic interpretation of the theory. In this note, the general condition imposed on transition amplitudes by the restriction of unitarity is explicitly stated. When applied to a theorem about tests of reciprocity originally given by Khalfin [11], one obtains not only a simpler and more direct proof of the theorem but also a stipulation on the nature of the variation whose occurrence Khalfin could infer, but

not specify further. The present formulation of the unitarity conditions could be used to 
explicitly take account of this constraint in possible future attempts to improve on the Weisskopf-Wigner approximation.

Section II presents the unitarity conditions which the exact transition amplitudes must satisfy, and shows how these lead to useful results, in addition to providing a new and simpler proof of Khalfin's theorem. Section III summarizes our conclusions.

\section{Unitarity Constraint on Transition Amplitudes}

Theorem. If $A_{k j}(t)$ is the exact transition amplitude for a state initially prepared in the state $j$ to be found in the state $k$ after a lapse of time $t$, unitarity requires that

$$
A_{j k}^{*}(-t)=A_{k j}(t)
$$

Correspondingly, if $f_{k j}(t)=A_{k j}(t) / A_{j k}(t)$, the function $f_{k j}(t)$ should satisfy the relation

$$
f_{k j}^{*}(-t) f_{k j}(t)=1
$$

Proof. By general principles of quantum mechanics, (using units $\hbar=1$ )

$$
A_{k j}(t)=\langle k|\exp (-i H t)| j\rangle
$$

for any two states $k$ and $j$, where $H$ is the complete Hamiltonian governing the timeevolution of the system.

Hermiticity of $H$ assures that the operator $U(t) \equiv \exp (-i H t)$ is unitary since $[\exp (-i H t)]^{\dagger}=\exp (i H t)$. Thus

$$
\langle k|U| j\rangle \equiv\langle k|\exp (-i H t)| j\rangle=\left\langle j\left|U^{\dagger}\right| k\right\rangle^{*}=\langle j|\exp (+i H t)| k\rangle^{*}
$$

From Eq. (3), the conjugated quantity on the R.H.S. is just $A_{j k}(-t)$. Consequently,

$$
A_{k j}(t)=A_{j k}^{*}(-t)
$$

for transitions induced by any Hermitian Hamiltonian $H$, which is exactly Eq. (1). This may be regarded as the unitarity constraint on transition amplitudes. If we rewrite Eq. (11) in the form:

$$
\phi_{j k} \equiv \frac{A_{j k}^{*}(-t)}{A_{k j}(t)}=1 \text {, }
$$


which must be valid for any $j, k$, then the condition $\phi_{j k}=\phi_{k j}$ leads to

$$
\frac{A_{j k}^{*}(-t)}{A_{k j}(t)}=\frac{A_{k j}^{*}(-t)}{A_{j k}(t)}
$$

which is equivalent to Eq. (2).

Q.E.D.

Our first application of the theorem will be to use it to show that the decay probability of an unstable state must be an even function of time. Setting $j=k$ in Eq. (四), we obtain

$$
P_{j j}(-t)=\left|A_{j j}(-t)\right|^{2}=\left|A_{j j}^{*}(t)\right|^{2}=P_{j j}(t)
$$

Therefore, the probability for a quantum system to remain in its initial state, and consequently also the complementary probability to make transitions to other states, must be an even function of time. This result has been known for some time, even though it has not yet found its way into many textbooks. The symmetry of $P_{j j}$ under $t \rightarrow-t$ is even more apparent if one writes

$$
P_{j j}(t)=A_{j j}^{*}(t) A_{j j}(t)=A_{j j}(-t) A_{j j}(t)
$$

making use of Eq. (1). Provided that $P_{j j}(t)$ is differentiable - a condition which is assured if $\langle H\rangle$ exists for the given initial state - at $t=0$, it follows that $\dot{P}_{j j}(t)$, which must correspondingly be an odd function of time, must vanish at $t=0$. We can explicitly verify this by calculating

$$
P_{k j}(t)=\left|A_{k j}(t)\right|^{2}=A_{k j}^{*}(t) A_{k j}(t)=A_{j k}(-t) A_{k j}(t)
$$

Then

$$
\dot{P}_{k j}(t)=\dot{A}_{j k}(-t) A_{k j}(t)+A_{j k}(-t) \dot{A}_{k j}(t)
$$

and, if we substitute the explicit expressions from Eq. (3), we obtain

$$
\dot{P}_{k j}(t)=i\left\langle j\left|H e^{i H t}\right| k\right\rangle A_{k j}(t)-i A_{k j}^{*}(t)\left\langle k\left|H e^{-i H t}\right| j\right\rangle
$$

and thus

$$
\dot{P}_{k j}(0)=2 \Im m(\langle j \mid k\rangle\langle k|H| j\rangle)
$$

which yields [12]

$$
\sum_{k} \dot{P}_{k j}(0)=2 \Im m\langle j|H| j\rangle=0
$$


This constraint has been called [13] the Quantum Zeno Effect. Observation [5] of the expected non-linear time-dependence at short times, for the closely related process of induced transitions, can be regarded as evidence in support of the effect.

A corollary statement is that, $\operatorname{since} \exp (-\gamma t)$ does not have a vanishing derivative at $t=0$, the hypothesis of exponentially decaying states is inconsistent with the requirement of unitarity. The deviations from exponential decay, both at very short times and at very long times, have been extensively studied by many authors [2, ㄱ.

Our next use of the theorem will be to provide a new and simpler proof of Khalfin's theorem [11]: that if the ratio of the transition amplitudes for two reciprocal [14] transitions $a \rightarrow b$ and $b \rightarrow a$ is constant, then the only possible value for the modulus $R$ of that constant is unity. We have seen that $f_{k j}(t)$, defined after Eq. (1), must satisfy

$$
f_{k j}(t) f_{k j}^{*}(-t)=1
$$

Thus, if we are given that $f_{k j}(t)=\zeta=$ constant for all $t \geq 0$, it follows from above that $f_{k j}^{*}$ must also be constant for all $t \leq 0$. Continuity of $\left|f_{k j}\right|$ at $t=0$ requires that

$$
R \equiv|\zeta|=1
$$

which is Khalfin's theorem. Since Khalfin arrived at this conclusion by a more complicated argument, we should like to note that the proof presented here required little [15 more than the assumption of unitarity. In particular, no assumption is required about the positivity of the spectrum of $H$, viz. the assumption Spec $H \geq 0$, made in Khalfin's proof, appears to be unnecessary.

Our next application of these ideas will be to prove that reciprocity must hold (independent of the question of time-reversal invariance) at very short times, as a consequence of unitarity alone. From Eq. (1),

$$
P_{j k}(0)=\left|A_{j k}(0)\right|^{2}=\left|A_{k j}^{*}(0)\right|^{2}=P_{k j}(0),
$$

which states that a kind of reciprocity is exactly valid at $t=0$. This can be understood directly as follows. For small values of $t$, let us expand the RHS of Eq. (3) in a power series,

$$
A_{k j}(t)=\langle k|1-i H t+\cdots| j\rangle
$$


If the state $k$ is not orthogonal to $j$, the value of the RHS of Eq. (14), for $t=0$, is the complex conjugate of, and therefore has the same magnitude as, $\langle j \mid k\rangle$. Therefore, $P_{k j}(0)$ and $P_{j k}(0)$ must be equal in that case. If $k$ is orthogonal [16] to $j$, we must go to the term linear in $t$ in Eq. (14), and we find that the transition amplitude is proportional to $H_{k j}$. But, since $H$ must be Hermitian, this is the complex conjugate of the matrix-element $H_{j k}$ for the inverse transition and we recover the result, reported in many textbooks [17], that in lowest-order perturbation theory, reciprocity follows from the Hermiticity of the interaction Hamiltonian. This requirement of reciprocity, independent of the $T$-invariance or otherwise of the Hamiltonian $H$, at early times $t \rightarrow 0$, can be stated more precisely by expanding the $P_{k j}(t)$ as a power series in $t$ :

$$
P_{k j}(t)=P_{k j}(0)+\dot{P}_{k j}(0) t+\frac{1}{2} \ddot{P}_{k j}(0) t^{2}+\mathcal{O}\left(t^{3}\right)
$$

We have already seen that $P_{k j}(0)=|\langle k \mid j\rangle|^{2}$ and $\dot{P}_{k j}(0)$, Eq. (10), both vanish if $\langle k \mid j\rangle=0$. By direct calculation, we find

$$
\ddot{P}_{k j}(0)=2|\langle k|H| j\rangle|^{2}-2 \Re e\left[\langle j \mid k\rangle\left\langle k\left|H^{2}\right| j\right\rangle\right]
$$

which shows explicitly that reciprocity must be preserved if $\langle k \mid j\rangle=0$, to order $t^{2}$, solely as a consequence of the Hermiticity of $H$, viz. of the requirement of unitarity for the time-evolution operator.

By an application of this result to the argument which led to the "quantum Zeno's paradox", we can conclude that any departure from reciprocity, which would be expected if $T$-invariance is not a symmetry of the underlying Hamiltonian, will be reduced or suppressed if the system undergoing change is monitored too closely. Thus, for example, frequent observation, amounting to a measurement of its strangeness, of a neutral kaon state, could reduce the inferred value of the $C P$ - and $T$-violating parameter $\varepsilon$ (under the assumption of TCP-invariance) relative to the one measured for "free" kaons. Possible implications of the corresponding quantum Zeno effect for baryogenesis in the Universe will be discussed elsewhere.

From his theorem Khalfin could conclude that, if reciprocity is not satisfied, $R$ must vary with time although nothing further could be said about the nature of that variation. The general solution for a function satisfying the unitarity condition (2), can be written 
as:

$$
f_{j k}(t)=\exp [g(t)+i h(t)]
$$

where $g(t)$ and $h(t)$ are real functions of $t$ which must be odd and even, respectively, under $t \rightarrow-t$. Any phenomenological representation of $A_{j k}(t)$, and correspondingly of $f_{j k}(t)$, to take account of possible deviations from reciprocity, which conforms to Eq. (17), will automatically satisfy the requirement of unitarity. The Weisskopf-Wigner formalism, as extended to the case of interfering [9] decaying states, was applied by Lee, Oehme, and Yang 18 to the $K^{0}-\bar{K}^{0}$ system, -in a form which can accommodate possible $T$ noninvariance-, and appears to adequately represent the data obtained thus far. Notwithstanding its great success, our foregoing discussion has shown that this description is not strictly compatible with unitarity of the exact theory [19].

Knowledge of the spectral content of the initial state, and thereby of the spectrum of $H$, determines [21, in principle, the complete time-evolution of the system through decomposition of its state-vector into a complete set of eigenvectors of $H$. Even in the absence of such detailed knowledge, any additional information about the spectrum of $H$, which could be expressed as further constraints [22] on the functions $g(t)$ and $h(t)$-beyond the conditions on $g(t)$ mentioned already - would obviously help to define the admissible forms of time-dependence.

We have already seen above that the Weisskopf-Wigner exponential Ansatz cannot exactly satisfy unitarity. Eq. ([17), with possible supplementary conditions, offers a natural point of departure for a new phenomenology satisfying exact unitarity.

\section{Conclusions}

In this note, we have shown that the existence of certain puzzling and unexpected phenomena, such as the quantum Zeno effect or deviations from Rutherford's law of exponential decay, can be directly traced back to the unitarity condition, which is required in quantum theory for a consistent description of any (isolated) dynamical system. Further consequences which are expected in principle, in addition to the Khalfin theorem mentioned already, include an analogue of the Zeno effect for the comparison of rates of reciprocal 
transitions. Whereas these rates are not directly related unless $T$-invariance is imposed on all relevant interactions, unitarity alone requires that a test of reciprocity must yield a result conforming to the $T$-invariant expectation if the measurements are made sufficiently rapidly. This means, for example, that even if we accept the usual interpretation [6] that the observed $C P$-noninvariance observed in neutral K-meson decays is associated with a $T$-noninvariant interaction, the corresponding expected [7] departure from reciprocity in $K^{0} \rightleftharpoons \bar{K}^{0}$ transitions would be suppressed, and indeed disappear, if the comparison were made at shorter and shorter times. Such asymmetries have been invoked 23] to explain the observed baryon asymmetry of the Universe; possible implications of this " $C P$ and $T$ quantum Zeno effect" will be discussed elsewhere.

\section{Acknowledgments}

One of us (PK) thanks H.M. Chan and F.E. Close for kind hospitality at the RutherfordAppleton Laboratory, and X. Tata for helpful comments. This research is supported in part by the U.S. Department of Energy.

\section{References}

[1] V. Weisskopf and E. Wigner, Z. Phys. 63, 54 (1930); ibid. 65, 18 (1930); for a compact account, see W. Heitler, Quantum Theory of Radiation, Oxford U.P., 3rd. ed.,1954.

[2] L. Khalfin, Zh. Eksper. Teor. Fiz. 33, 1371 (1957) [Sov. Phys. JETP 6, 1053 (1958)].

[3] E.g., J. Schwinger, Ann. Phys. 9, 169 (1958).

[4] C.B. Chiu, E.C.G. Sudarshan, and B. Misra, Phys. Rev. D16, 520 (1977); C. Bernardini, L. Maiani, and M. Testa, Phys. Rev. Lett. 712687 (1993); G. García-Calderón, J.L. Mateos, and M. Moshinsky, Phys. Rev. Lett. 74, 337 (1995).

[5] W.M. Itano, D.J. Heinzen, J.J. Bollinger, and D.J. Wineland, Phys. Rev. A41, 2295 (1990). 
[6] V.L. Fitch, Rev. Mod. Phys. 53, 367 (1981).

[7] P.K. Kabir, Phys. Rev. D2, 540 (1970).

[8] A. Pilaftsis, Z. Phys. C47, 95 (1990); J. Papavassiliou and A. Pilaftsis, Gauge Invariance and Unstable Particles, Rutherford report (1995), RAL-TR-95-021, Phys. Rev. Lett. (to appear).

[9] G. Breit and I.S. Lowen, Phys. Rev. 46, 590 (1934).

[10] E. Rutherford and F. Soddy, Phil. Mag. 6, 576 (1903).

[11] L. Khalfin, unpublished Univ. of Texas Report DOE-ER 40200-211, 1990, cited by C.B. Chiu and E.C.G. Sudarshan, Phys. Rev. D42, 3712 (1990).

[12] Strictly speaking, the initial state $j$ should be excluded from the sum over final states $k$. The RHS of Eq. (11) is not affected by this restriction. This confirms the result of the direct calculation of $\dot{P}_{j j}(0)$ in Ref. [13].

[13] B. Misra and E.C.G. Sudarshan, J. Math. Phys. 18, 756 (1977).

[14] For the present, following Khalfin, we restrict our discussion to states $a, b$ which can be transformed into the corresponding "time-reversed" states $a_{T}, b_{T}$ by rotation and/or other symmetry of the governing Hamiltonian $H$.

[15] To assure the existence of the transition rates, it would suffice, for example, if the initial and final states had finite expectation values of $\langle H\rangle$.

[16] A convenient way to think of this is to imagine that $k$ and $j$ are distinct stationary states of some Hamiltonian $H_{0}$ and that transitions between them are induced by $H^{\prime}=H-H_{0}$.

[17] E.g., J.J. Sakurai, Modern Quantum Mechanics, Addison-Wesley, New York, 1992.

[18] T.D. Lee, R. Oehme, and C.N. Yang, Phys. Rev. 106, 340 (1957).

[19] Bell's proof [20], that unitarity can be satisfied in a generalized Weisskopf-Wigner theory, applies to a system whose time-evolution is exactly determined by a nonHermitian (time-independent) effective Hamiltonian, viz. whose "eigenstates" decay 
exponentially. Since we have seen that truly exponential decay cannot occur for any admissible (Hermitian) Hamiltonian, Bell's demonstration does not carry over to the exact (complete) theory.

[20] J.S. Bell, in "High Energy Physics", C. de Witt and M. Jacob, eds., Gordon \& Breach, New York, 1965.

[21] N.S. Krylov and V.A. Fock, Zh. Eksp. Teor. Fiz. 17, 93 (1947).

[22] Apart from the requirements of unitarity, further restrictions may follow from other physical requirements, L.A. Khalfin, private communication.

[23] A.D. Sakharov, Pis'ma Zh. Eksp. Teor. Fiz. 5, 32 (1967), [JETP Letters 5, 24 (1967)]. 